\title{
TAUBERIAN THEORY FOR MULTIVARIATE REGULARLY VARYING DISTRIBUTIONS WITH APPLICATION TO PREFERENTIAL ATTACHMENT NETWORKS
}

\author{
SIDNEY RESNICK AND GENNADY SAMORODNITSKY
}

\begin{abstract}
Abel-Tauberian theorems relate power law behavior of distributions and their transforms. We formulate and prove a multivariate version for non-standard regularly varying measures on $\mathbb{R}_{+}^{p}$ and then apply it to prove that the joint distribution of in- and out-degree in a directed edge preferential attachement model has jointly regularly varying tails.
\end{abstract}

\section{INTRODUCTION}

This paper has two themes: (i) Abel-Tauberian theorems relate power law behavior of distributions and their transforms. (ii) Such Abel-Tauberian theorems can be used to study power law behavior of in- and out-degree of directed edge preferential attachment network models.

Abel-Tauberian theorems relate regular variation of infinite Radon measures $U(\boldsymbol{x})=U([\mathbf{0}, \boldsymbol{x}])$ on $\mathbb{R}_{+}^{p}$ to regular variation of their Laplace transforms

$$
\hat{U}(\mathbf{1} / \boldsymbol{x})=\int_{\mathbb{R}_{+}^{p}} e^{-\sum_{i=1}^{p} s_{i} / x_{i}} U(d \boldsymbol{s}), \quad \boldsymbol{x}>\mathbf{0} .
$$

In one dimension when $p=1$, such theorems provide standard tools for obtaining asymptotic power law tails for cases when a probability description is more easily specified by the transform rather than the distribution. Often the transform rather than the distribution is accessible as a solution to difference or recursive relations. Application areas include queueing theory, branching processes, insurance modeling and network analysis. Standard references covering the essentials in one dimension are Bingham et al. (1987), Feller (1971). Transform theory when $p>1$ for the standard case of regular variation are considered in Resnick (1991, 2007), Stadtmüller (1981), Stadtmüller and Trautner (1979, 1981), Stam (1977), Yakimiv (2005). In this paper we consider an Abel-Tauberian theorem for the non-standard case of regular variation where scaling functions for different components have different tail indices.

Preferential attachment is an important mechanism for describing growth of directed networks where a new node attaches to an existing node or new edges are created according to probabilistic postulates that take into account the in- and out-degree of the existing nodes. We consider models studied by Bollobás et al. (2003) and Krapivsky and Redner (2001). Based on solutions to difference equations, Samorodnitsky et al. (2014) derived the joint generating function of limiting frequencies for in-degree and out-degree. In this paper we explain how the joint non-standard regular variation of in- and out-degree can be obtained from the joint generating function using Abel-Tauberian theory.

1991 Mathematics Subject Classification. Primary 60G70, 05C80.

Key words and phrases. multivariate heavy tails, preferential attachment model, scale free networks, Tauberian theory.

This research was supported by the ARO MURI grant W911NF-12-10385 to Cornell University. 
This paper is organized as follows. We start with a brief summary of multivariate regular variation of measures in Section 2 to establish notation and basic concepts. Section 3 gives the Abel-Tauberian theorem for measures which are non-standard regularly varying. In Section 4.3, we apply the Tauberian theory to study the multivariate power laws of in- and out-degree in the preferential attachment model. Section 4.1 includes a detailed description of the preferential attachment model, Section 4.2 summarizes known results about the joint generating function of in- and out-degree and Section 4.3 applies the Tauberian theory to obtain the joint power law behavior of in- and out-degree.

\section{Multivariate Regular VARiation}

We briefly review the basic concepts of multivariate regular variation which forms the mathematical framework for multivariate heavy tails. We emphasize two dimensions since this is the context for the application to in- and out-degree but generally the extension to $p \geq 2$ dimensions is clear. See Resnick (2007) for more detail.

A random vector $(X, Y) \geq \mathbf{0}$ has a distribution that is non-standard regularly varying if there exist scaling functions $a(t) \uparrow \infty$ and $b(t) \uparrow \infty$ and a non-zero limit measure $\nu(\cdot)$ called the limit or tail measure such that as $t \rightarrow \infty$,

$$
t P[(X / a(t), Y / b(t)) \in \cdot] \stackrel{v}{\rightarrow} \nu(\cdot)
$$

where " $\stackrel{v}{\rightarrow}$ " denotes vague convergence of measures in $M_{+}\left([0, \infty]^{2} \backslash\{\mathbf{0}\}\right)=M_{+}(\mathbb{E})$, the space of Radon measures on $\mathbb{E}$. The scaling functions will be regularly varying and we assume their indices are positive and therefore, without loss of generality, we may suppose $a(t)$ and $b(t)$ are continuous and strictly increasing. The phrasing in (2.1) implies the marginal distributions have regularly varying tails.

In case $a(t)=b(t),(X, Y)$ has a distribution with standard regularly varying tails. Given a vector with a distribution which is non-standard regularly varying, there are at least two methods for standardizing the vector so that the transformed vector has standard regular variation (Resnick, 2007, Section 9.2.3). The simplest is the power method which is justified when the scaling functions are power functions:

$$
a(t)=t^{1 / \gamma_{1}}, \quad b(t)=t^{1 / \gamma_{2}}, \quad \gamma_{i}>0, i=1,2 .
$$

For instance with $c=\gamma_{1} / \gamma_{2}$,

$$
t P\left[\left(X^{c} / t^{1 / \gamma_{2}}, Y / t^{1 / \gamma_{2}}\right) \in \cdot\right] \stackrel{v}{\rightarrow} \tilde{\nu}(\cdot),
$$

where if $T(x, y)=\left(x^{c}, y\right)$, then $\tilde{\nu}=\nu \circ T^{-1}$. Since the two scaling functions in (2.2) are the same, the regular variation is now standard. The measure $\tilde{\nu}$ will have a scaling property and if the coordinate system is changed properly, $\tilde{\nu}$ will disintegrate to a product; for example the polar coordinate transform is one such coordinate system change achieving the disintegration into a product and this provides access to an angular measure that is one way to describe the asymptotic dependence structure of the standardized $(X, Y)$.

The non-standard regular variation of Radon measures is defined in (3.4) below. 
2.1. Miscellaneous notation. Here is a notation and concept summary.

$R V_{\beta} \quad$ Regularly varying functions with index $\beta>0$. We pick versions of such functions that are continuous and strictly increasing.

$M_{+}(\mathbb{E}) \quad$ Radon measures on $\mathbb{E}:=[0, \infty]^{p} \backslash\{\mathbf{0}\}$ metrized by vague convergence.

$M_{+}\left(\mathbb{R}_{+}^{p}\right)$ Radon measures on $\mathbb{R}_{+}^{p}$ metrized by vague convergence.

$\stackrel{v}{\rightarrow} \quad$ Vague convergence in $M_{+}\left(\mathbb{R}_{+}^{p}\right)$ or $M_{+}(\mathbb{E})$ as appropriate.

$\boldsymbol{x} \quad \boldsymbol{x}=\left(x_{1}, \ldots, x_{p}\right)$.

$\boldsymbol{\lambda} \boldsymbol{x} \quad\left(\lambda_{1} x_{1}, \ldots, \lambda_{p} x_{p}\right)$.

$\boldsymbol{\lambda}^{\prime} \boldsymbol{x} \quad \sum_{i=1}^{p} \lambda_{i} x_{i}$.

$1 \quad \mathbf{1}=(1, \ldots, 1)$.

$\mathbf{0} \quad \mathbf{0}=(0, \ldots, 0)$.

$\hat{U} \quad$ Laplace transform of a measure $U ; \hat{U}(\boldsymbol{\lambda})=\int_{\mathbb{R}_{+}^{p}} \exp \left\{-\boldsymbol{\lambda}^{\prime} \boldsymbol{x}\right\} U(d \boldsymbol{x})$.

$\aleph \quad \aleph=\left\{\boldsymbol{x} \in \mathbb{R}_{+}^{p}:\|\boldsymbol{x}\|=1\right\}$, the unit sphere in $\mathbb{R}_{+}^{p}$ for some norm $\|\cdot\|$.

In general vectors are denoted by bold letters, eg. $\boldsymbol{x}=\left(x_{1}, \ldots, x_{p}\right)$. Operations on vectors, unless noted otherwise, should be interpreted componentwise. Thus, $\boldsymbol{\lambda} \boldsymbol{x}=\left(\lambda_{1} x_{1}, \ldots, \ldots, \lambda_{p} x_{p}\right)$ but (as noted) $\boldsymbol{\lambda}^{\prime} \boldsymbol{x}=\sum_{i=1}^{p} \lambda_{i} x_{i}$. Also $[\mathbf{0}, \boldsymbol{x}]=\left\{\left(u_{1}, \ldots, u_{p}\right): 0 \leq u_{i} \leq x_{i}, i=1, \ldots, p\right\}$.

\section{A TAUBERIAN THEOREM FOR NONSTANDARD REGULAR VARIATION}

In this section we give an Abel-Tauberian theorem which relates non-standard regular variation of a Radon measure $U(\boldsymbol{x})$ on $\mathbb{R}_{+}^{p}$ to non-standard regular variation of the Laplace transform $\hat{U}(\mathbf{1} / \boldsymbol{x})$. Versions in the standard case when $p>1$ are considered in Resnick (1991, 2007), Stadtmüller (1981), Stadtmüller and Trautner (1979, 1981), Stam (1977), Yakimiv (2005).

3.1. Assumptions. For $p \geq 1$, suppose $U$ is a measure on $\mathbb{R}_{+}^{p}$ satisfying

$$
\hat{U}(\boldsymbol{\lambda}):=\int_{\mathbb{R}_{+}^{p}} e^{-\boldsymbol{\lambda}^{\prime} \boldsymbol{x}} U(d \boldsymbol{x})<\infty, \quad \boldsymbol{\lambda}>\mathbf{0} .
$$

This implies $U$ is Radon on $\mathbb{R}_{+}^{p}$ since for $\boldsymbol{\lambda}>\mathbf{0}$, and $\boldsymbol{y}>\mathbf{0}$,

$$
\begin{aligned}
\infty & >\int_{\mathbb{R}_{+}^{p}} e^{-\boldsymbol{\lambda}^{\prime} \boldsymbol{x}} U(d \boldsymbol{x}) \geq \int_{\mathbb{R}_{+}^{p}} e^{-\boldsymbol{\lambda}^{\prime} \boldsymbol{x}} 1_{[\mathbf{0}, \boldsymbol{y}]}(\boldsymbol{x}) U(d \boldsymbol{x}) \\
& \geq e^{-\boldsymbol{\lambda}^{\prime} \boldsymbol{y}} \int 1_{[\mathbf{0}, \boldsymbol{y}]}(\boldsymbol{x}) U(d \boldsymbol{x})=e^{-\boldsymbol{\lambda}^{\prime} \boldsymbol{y}} U([\mathbf{0}, \boldsymbol{y}]) .
\end{aligned}
$$

So $U(\boldsymbol{y}):=U([\mathbf{0}, \boldsymbol{y}])<\infty$ for $\boldsymbol{y}>\mathbf{0}$ and therefore $U \in M_{+}\left(\mathbb{R}_{+}^{p}\right)$.

For $i=1, \ldots, p$, assume

$$
b_{i}(t) \in R V_{1 / \gamma_{i}}, \gamma_{i}>0, \quad i=1, \ldots, p .
$$

Write $\boldsymbol{b}(t)=\left(b_{1}(t), \ldots, b_{p}(t)\right)$ and $\gamma=\left(\gamma_{1}, \ldots, \gamma_{p}\right)$. Set

$$
U_{t}(\boldsymbol{x})=\frac{1}{t} U(\boldsymbol{b}(t) \boldsymbol{x}) .
$$


3.2. Regular variation of the measure implies regular variation of the transform. For this section assume $U$ satisfies (3.1) and $U_{t}$ is defined in (3.3). The scaling functions $b_{i}(t)$ satisfy (3.2). The non-standard regular variation assumption for $U$ is that there exist $U_{\infty} \in M_{+}\left(\mathbb{R}_{+}^{p}\right)$, $U_{\infty} \not \equiv 0$, such that

$$
U_{t} \stackrel{v}{\rightarrow} U_{\infty}, \quad \text { in } M_{+}\left(\mathbb{R}_{+}^{p}\right) .
$$

If we can choose the scaling functions $\left(b_{i}, i=1, \ldots, p\right)$ to be identical, then the regular variation is standard.

3.2.1. Consequences. The assumptions have consequences needed for further work.

1. Continuous convergence: The convergence in (3.4) is continuous convergence on $(\mathbf{0}, \infty):=$ $(0, \infty)^{p}$; that is, if as $t \rightarrow \infty, \boldsymbol{x}(t) \rightarrow \boldsymbol{x}(\infty) \in(\mathbf{0}, \infty)$, then

$$
U_{t}(\boldsymbol{x}(t)) \rightarrow U_{\infty}(\boldsymbol{x}(\infty)), \quad(t \rightarrow \infty)
$$

provided $\boldsymbol{x}(\infty)$ is a continuity point of $U_{\infty}(\boldsymbol{x})$. This is a monotonicity argument: If $\boldsymbol{x}(\infty)$ and $\boldsymbol{x}(\infty)+\epsilon \mathbf{1}$ are continuity points of $U_{\infty}(\boldsymbol{x})$, then

$$
\begin{aligned}
\limsup _{t \rightarrow \infty} U_{t}(\boldsymbol{x}(t)) & \leq \lim _{t \rightarrow \infty} U_{t}(\boldsymbol{x}(\infty)+\epsilon \mathbf{1}) \\
& =U_{\infty}(\boldsymbol{x}(\infty)+\epsilon \mathbf{1}),
\end{aligned}
$$

and letting $\epsilon \downarrow 0$ in such a way that $\boldsymbol{x}(\infty)+\epsilon \mathbf{1}$ are continuity points of $U_{\infty}(\boldsymbol{x})$ yields

$$
\limsup _{t \rightarrow \infty} U_{t}(\boldsymbol{x}(t)) \leq U_{\infty}(\boldsymbol{x}(\infty))
$$

A reverse inequality is obtained similarly. A consequence of the continuous convergence is the scaling property: for $c>0$

$$
U_{\infty} \circ T_{c}^{-1}=c U_{\infty}
$$

where $T_{c}: \mathbb{R}_{+}^{p} \rightarrow \mathbb{R}_{+}^{p}$ is defined by $T_{c} \boldsymbol{x}=c^{-1 / \gamma} \boldsymbol{x}$. It is enough to check that for $\boldsymbol{x}>0$

$$
U_{\infty}\left(c^{1 / \gamma} \boldsymbol{x}\right)=c U_{\infty}(\boldsymbol{x}) .
$$

Indeed,

$$
U_{\infty}\left(c^{\mathbf{1} / \gamma} \boldsymbol{x}\right)=\lim _{t \rightarrow \infty} \frac{1}{t} U\left(b_{1}(t) c^{1 / \gamma_{1}} x_{1}, \ldots, b_{p}(t) c^{1 / \gamma_{p}} x_{p}\right)
$$

and by continuous convergence, this is

$$
\begin{aligned}
& =\lim _{t \rightarrow \infty} c \frac{1}{c t} U\left(b_{1}(c t)\left(\frac{b_{1}(t)}{b_{1}(c t)} c^{1 / \gamma_{1}}\right) x_{1}, \ldots, b_{p}(c t)\left(\frac{b_{p}(t)}{b_{p}(c t)} c^{1 / \gamma_{p}}\right) x_{p}\right) \\
& =c U_{\infty}(\boldsymbol{x}) .
\end{aligned}
$$

The scaling property implies, in particular, that all points $\boldsymbol{x}$ are continuity points of $U_{\infty}$. 
2. Laplace transform of $U_{\infty}$ exists: Let $i_{*} \in\{1, \ldots, p\}$ be such that $\gamma_{i_{*}} \geq \gamma_{i}$ for all $i \in\{1, \ldots, p\}$. It follows from the scaling property (3.6) that for any $a>0$

$$
U_{\infty}\left(\left\{\boldsymbol{x}: \sum_{i=1}^{p} x_{i} \leq a\right\}\right) \leq a^{\gamma_{i *}} U_{\infty}\left(\left\{\boldsymbol{x}: \sum_{i=1}^{p} x_{i} \leq 1\right\}\right) .
$$

Therefore, for $\boldsymbol{\lambda}>\mathbf{0}$,

$$
\begin{gathered}
\hat{U}_{\infty}(\boldsymbol{\lambda}) \leq \int_{\mathbb{R}_{+}^{p}} e^{-\min _{i} \lambda_{i} \sum_{i} x_{i}} U_{\infty}(d \boldsymbol{x}) \\
\leq U_{\infty}\left(\left\{\boldsymbol{x}: \sum_{i=1}^{p} x_{i} \leq 1\right\}\right) \int_{0}^{\infty} e^{-\left(\min _{i} \lambda_{i}\right) x} \gamma_{i_{*}} x^{\gamma_{i *}-1} d x<\infty .
\end{gathered}
$$

3.2.2. The result. This section requires a regularity condition: for any $\boldsymbol{x}>\mathbf{0}$,

$$
\lim _{y \rightarrow \infty} \limsup _{t \rightarrow \infty} \int_{\cup_{i=1}^{p}\left[v_{i}>y\right]} e^{-\sum_{i=1}^{p} v_{i} / x_{i}} U_{t}(d \boldsymbol{v})=0 .
$$

Proposition 1. Assume (3.2) and suppose that $U$ satisfies the non-standard regular variation condition (3.4). Then the Laplace transforms $\hat{U}(\mathbf{1} / \boldsymbol{x})$ and $\hat{U}_{\infty}(\mathbf{1} / \boldsymbol{x})$ are distribution functions of Radon measures on $\mathbb{R}_{+}^{p}$ and these measures inherit the non-standard regular variation: for $\boldsymbol{x}>0$

$$
\frac{1}{t} \hat{U}(\mathbf{1} /(\boldsymbol{b}(t) \boldsymbol{x})) \rightarrow \hat{U}_{\infty}(\mathbf{1} / \boldsymbol{x}),
$$

provided (3.9) also holds.

Proof. Let $E_{1}, \ldots, E_{p}$ be iid standard exponentially distributed random variables so that

$$
\mathcal{F}=\left(\frac{1}{E_{1}}, \ldots, \frac{1}{E_{p}}\right)
$$

are iid standard Frechét random variables with marginal distribution

$$
P\left[1 / E_{1} \leq x\right]=e^{-1 / x}, \quad x>0 .
$$

From (3.4) we get

$$
P[\mathcal{F} \in \cdot] \times U_{t} \stackrel{v}{\rightarrow} P[\mathcal{F} \in \cdot] \times U_{\infty}
$$

in $M_{+}\left([0, \infty]^{p} \times \mathbb{R}_{+}^{p}\right)$. Define $h:[0, \infty]^{p} \times \mathbb{R}_{+}^{p} \mapsto[0, \infty]^{p} \times \mathbb{R}_{+}^{p}$ by

$$
h(\boldsymbol{x}, \boldsymbol{y})=(\boldsymbol{x} \boldsymbol{y}, \boldsymbol{y}),
$$

where $\boldsymbol{x} \boldsymbol{y}=\left(x_{i} y_{i}, i=1, \ldots, p\right)$ is componentwise multiplication, and we set $0 \cdot \infty=0$. The map $h$ satisfies the compactness condition of (Resnick, 2007, Proposition 5.5): Suppose $A \subset[0, \infty]^{p} \times \mathbb{R}_{+}^{p}$ satisfies the condition that there exists $M>0$ such that

$$
(\boldsymbol{x}, \boldsymbol{y}) \in A \quad \text { implies } \bigvee_{i=1}^{p} y_{i} \leq M
$$

Then

satisfies

$$
h^{-1}(A)=\{(\boldsymbol{u}, \boldsymbol{v}):(\boldsymbol{u} \boldsymbol{v}, \boldsymbol{v}) \in A\}
$$

$$
(\boldsymbol{x}, \boldsymbol{y}) \in h^{-1}(A) \quad \text { implies } \bigvee_{i=1}^{p} y_{i} \leq M
$$


Thus if $A$ is relatively compact, so is $h^{-1}(A)$. Therefore (3.11) and (Resnick, 2007, Proposition 5.5) imply

$$
\left(P[\mathcal{F} \in \cdot] \times U_{t}\right) \circ h^{-1} \stackrel{v}{\rightarrow}\left(P[\mathcal{F} \in \cdot] \times U_{\infty}\right) \circ h^{-1}, \text { in } M_{+}\left([0, \infty]^{p} \times \mathbb{R}_{+}^{p}\right) .
$$

Evaluate the left side of (3.12) on the relatively compact set $[\mathbf{0}, \boldsymbol{x}] \times[\mathbf{0}, y \mathbf{1}]$ (assuming $\boldsymbol{x}>\mathbf{0}$ and $y>0$ are chosen to make this is a continuity set of the limit measure) and we get,

$$
\begin{aligned}
(P[\mathcal{F} \in & \left.\cdot] \times U_{t}\right) \circ h^{-1}([\mathbf{0}, \boldsymbol{x}] \times[\mathbf{0}, y \mathbf{1}])=\iint_{\{(\boldsymbol{u}, \boldsymbol{v}): \boldsymbol{u} \boldsymbol{v} \leq \boldsymbol{x}, \boldsymbol{v} \leq y \mathbf{1}\}} P[\mathcal{F} \in d \boldsymbol{u}] U_{t}(d \boldsymbol{v}) \\
& =\int_{\boldsymbol{v} \leq y \mathbf{1}} \int_{\boldsymbol{u} \leq \boldsymbol{x} / \boldsymbol{v}} P[\mathcal{F} \in d \boldsymbol{u}] U_{t}(d \boldsymbol{v})=\int_{\boldsymbol{v} \leq y \mathbf{1}} \prod_{i=1}^{p} e^{-v_{i} / x_{i}} U_{t}(d \boldsymbol{v}) \\
& =\int_{\boldsymbol{v} \leq y \mathbf{1}} e^{-\sum_{i=1}^{p} v_{i} / x_{i}} U_{t}(d \boldsymbol{v})
\end{aligned}
$$

and applying (3.12) we conclude that as $t \rightarrow \infty$ this converges to

$$
\rightarrow \int_{\boldsymbol{v} \leq y \mathbf{1}} e^{-\sum_{i=1}^{p} v_{i} / x_{i}} U_{\infty}(d \boldsymbol{v}) .
$$

Now let $y \rightarrow \infty$ and apply monotone convergence to get the integral in (3.14) to converge to

$$
\rightarrow \int_{\mathbb{R}_{+}^{p}} e^{-\sum_{i=1}^{p} v_{i} / x_{i}} U_{\infty}(d \boldsymbol{v})=: \hat{U}_{\infty}(\mathbf{1} / \boldsymbol{x})
$$

So to show for $\boldsymbol{x}>\mathbf{0}$ that

$$
\hat{U}_{t}(\mathbf{1} / \boldsymbol{x}):=\int_{\mathbb{R}_{+}^{p}} e^{-\sum_{i=1}^{p} v_{i} / x_{i}} U_{t}(d \boldsymbol{v})=\frac{1}{t} \hat{U}(\mathbf{1} /(\boldsymbol{b}(t) \boldsymbol{x})) \rightarrow \hat{U}_{\infty}(\mathbf{1} / \boldsymbol{x}),
$$

we must verify that

$$
\lim _{y \rightarrow \infty} \limsup _{t \rightarrow \infty}\left|\int_{\boldsymbol{v} \leq y \mathbf{1}} e^{-\sum_{i=1}^{p} v_{i} / x_{i}} U_{t}(d \boldsymbol{v})-\int_{\mathbb{R}_{+}^{p}} e^{-\sum_{i=1}^{p} v_{i} / x_{i}} U_{t}(d \boldsymbol{v})\right|=0,
$$

which is (3.9).

The statement that $\hat{U}(\mathbf{1} / \boldsymbol{x})$ is a distribution function of a Radon measure follows from 3.1) since, as in (3.13),

$$
\infty>\hat{U}(\mathbf{1} / \boldsymbol{x})=\lim _{y \rightarrow \infty}(P[\mathcal{F} \in \cdot] \times U) \circ h^{-1}([\mathbf{0}, \boldsymbol{x}] \times[\mathbf{0}, y \mathbf{1}]),
$$

a limit of the distribution functions of a Radon measures. The statement about $\hat{U}_{\infty}(\mathbf{1} / \boldsymbol{x})$ follows similarly using the fact that $\hat{U}_{\infty}(\boldsymbol{\lambda})<\infty$ for $\boldsymbol{\lambda}>0$ by (3.8).

Rather than checking condition (3.9) directly, it may sometimes be easier to verify the following sufficient condition: for every $1 \leq i \leq p$, suppose

$$
U_{i}(x)=U\left(\mathbb{R}_{+} \times \cdots \times[0, x] \times \mathbb{R}_{+} \times \cdots \times \mathbb{R}_{+}\right)<\infty,
$$

and

$$
\lim _{t \rightarrow \infty} \frac{U_{i}\left(b_{i}(t) x\right)}{t}=x^{\gamma_{i}}, \quad x>0
$$


To see why these conditions are sufficient for (3.9), dominate the integral in (3.9) by

$$
\sum_{i=1}^{p} \int_{\left[v_{i}>y\right]} e^{-\sum_{i=1}^{p} v_{i} / x_{i}} U_{t}(d \boldsymbol{v})
$$

and focus, for simplicity, on the integral with $i=1$ which can be written as

$$
\begin{aligned}
\int_{\left[v_{1}>y\right]} & {\left[\prod_{l=1}^{p} \int_{s_{l}>v_{l}} \frac{1}{x_{l}} e^{-s_{l} / x_{l}} d s_{l}\right] U_{t}(d \boldsymbol{v}) } \\
& =\int_{s_{1}>y}\left(\int_{\substack{s_{l} \geq v_{l} ; l=2, \ldots, p \\
v_{1} \leq s_{1}}} U_{t}(d \boldsymbol{v})\right) \prod_{l=1}^{p} \frac{1}{x_{l}} e^{-s_{l} / x_{l}} d s_{1} \ldots d s_{p} \\
& =\int_{\boldsymbol{s} \in(y, \infty) \times \mathbb{R}_{+}^{p-1}} U_{t}\left(\left(y, s_{1}\right] \times\left[0, s_{2}\right] \times \ldots \times\left[0, s_{p}\right]\right) \prod_{l=1}^{p} \frac{1}{x_{l}} e^{-s_{l} / x_{l}} d s_{1} \ldots d s_{p} \\
& \leq \int_{s \in(y, \infty) \times \mathbb{R}_{+}^{p-1}} U_{t}(\boldsymbol{s}) \prod_{l=1}^{p} \frac{1}{x_{l}} e^{-s_{l} / x_{l}} d s_{1} \ldots d s_{p} \\
& \leq \int_{y}^{\infty} U_{t}\left(\left[0, s_{1}\right] \times \mathbb{R}_{+}^{p-1}\right) \frac{1}{x_{1}} e^{-s_{1} / x_{1}} d s_{1} \\
& =\int_{y}^{\infty} \frac{U_{1}\left(b_{1}(t) s_{1}\right)}{t} \frac{1}{x_{1}} e^{-s_{1} / x_{1}} d s_{1}
\end{aligned}
$$

and by an application of the Potter bounds, for given $\delta>0$ and large enough $t$ and $y>1$, the previous expression is bounded by

$$
\leq \int_{y}^{\infty}(\text { const }) s^{\gamma_{1}+\delta} \frac{1}{x_{1}} e^{-s_{1} / x_{1}} d s_{1} \rightarrow 0, \quad(y \rightarrow \infty) .
$$

3.3. Regular variation of the transform implies regular variation of the measure. In this section we assume (3.1), (3.2), (3.9) and additionally assume there exists a finite-valued function $\hat{U}_{\infty}$ such that for $\boldsymbol{x}>\mathbf{0}$,

$$
\frac{1}{t} \hat{U}(\mathbf{1} /(\boldsymbol{b}(t) \boldsymbol{x}))=\left(P[\mathcal{F} \in \cdot] \times U_{t}\right) \circ h^{-1}\left([\mathbf{0}, \boldsymbol{x}] \times \mathbb{R}_{+}^{p}\right) \rightarrow \hat{U}_{\infty}(\mathbf{1} / \boldsymbol{x}) .
$$

We claim that $\left\{U_{t}\right\}$ is a tight family of measures on $\mathbb{R}_{+}^{p}$. It suffices to show that for any $M>0$

$$
\sup _{t \geq 1} U_{t}[\mathbf{0}, M \mathbf{1}]<\infty .
$$

Given $\epsilon>0$, there exists $\delta>0$ such that $P\left[\mathcal{F} \in\left[\delta \mathbf{1}, \delta^{-1} \mathbf{1}\right]\right] \geq 1-\epsilon$. For $\boldsymbol{x}>\mathbf{0}$,

$$
\begin{aligned}
\hat{U}_{t}(\mathbf{1} / \boldsymbol{x}) & =\left(P[\mathcal{F} \in \cdot] \times U_{t}\right) \circ h^{-1}\left([\mathbf{0}, \boldsymbol{x}] \times \mathbb{R}_{+}^{p}\right) \\
& =\int_{\{(\boldsymbol{u}, \boldsymbol{v}): \boldsymbol{u} \boldsymbol{v} \leq \boldsymbol{x}\}} P[\mathcal{F} \in d \boldsymbol{u}] U_{t}(d \boldsymbol{v}) \geq \int_{\boldsymbol{u} \in\left[\delta \mathbf{1} \delta^{-1} \mathbf{1}\right]} P[\mathcal{F} \in d \boldsymbol{u}] U_{t}(d \boldsymbol{v}) \\
& =\int_{\boldsymbol{u} \in\left[\delta \mathbf{1}, \delta^{-1} \mathbf{1}\right]} U_{t}(\boldsymbol{x} / \boldsymbol{u}) P[\mathcal{F} \in d \boldsymbol{u}] \\
& \geq U_{t}\left(\boldsymbol{x} / \delta^{-1}\right) P\left[\mathcal{F} \in\left[\delta \mathbf{1}, \delta^{-1} \mathbf{1}\right]\right] \geq U_{t}\left(\boldsymbol{x} / \delta^{-1}\right)(1-\epsilon) .
\end{aligned}
$$


Set $\boldsymbol{x}=\delta^{-1} M \mathbf{1}$ and then

$$
\sup _{t \geq 1} U_{t}(M \mathbf{1}) \leq \frac{1}{1-\epsilon} \sup _{t \geq 1} \hat{U}_{t}\left(1 /\left(\delta^{-1} M \mathbf{1}\right)\right)<\infty
$$

by convergence in (3.18).

Suppose $\left\{U_{t_{n}}\right\}$ is a convergent subsequence, say $U_{t_{n}} \rightarrow L$ in $M_{+}\left(\mathbb{R}_{+}^{p}\right)$. Since we assume (3.9) holds, the mechanics of Section 3.2 .2 give for $\boldsymbol{x}>\mathbf{0}$,

$$
\hat{U}_{t_{n}}(\mathbf{1} / \boldsymbol{x}) \rightarrow \hat{L}(\mathbf{1} / \boldsymbol{x})<\infty, \quad\left(t_{n} \rightarrow \infty\right)
$$

at continuity points of the limit. From (3.18) we get $\hat{L}=\hat{U}_{\infty}$. If there are two subsequential limits $L_{1}, L_{2}$ of $\left\{U_{t}\right\}$ then $\hat{L}_{1}=\hat{L}_{2}=\hat{U}_{\infty}$ and so $\left\{U_{t}\right\}$ converges in $M_{+}\left(\mathbb{R}_{+}^{p}\right)$ to some $U_{\infty}$ with transform $\hat{U}_{\infty}$.

We summarize:

Proposition 2. Suppose $U \in M_{+}\left(\mathbb{R}_{+}^{p}\right)$ and let (3.1), (3.2), (3.9) hold. If there exists a finite-valued function $\hat{U}_{\infty}$ such that (3.18) holds, then (3.4) holds for some measure $U_{\infty} \in M_{+}\left(\mathbb{R}_{+}^{p}\right)$ whose Laplace transform is $\hat{U}_{\infty}$. Moreover,

$$
U_{t}(\boldsymbol{x})=\frac{1}{t} U(\boldsymbol{b}(t) \boldsymbol{x}) \rightarrow U_{\infty}(\boldsymbol{x}), \quad(t \rightarrow \infty)
$$

for all $\boldsymbol{x}$.

\section{Application to preferential attachment network models.}

4.1. Model description. The directed edge preferential attachement model studied by Krapivsky and Redner (2001) and Bollobás et al. (2003) is a model for a growing directed random graph. The dynamics of the model are as follows. Choose nonnegative real parameters $\alpha, \beta, \gamma, \delta_{\text {in }}$ and $\delta_{\text {out }}$, such that $\alpha+\beta+\gamma=1$. To avoid degenerate situations assume each of the numbers $\alpha, \beta, \gamma$ is strictly smaller than 1 .

At each step of the growth algorithm we add one edge to an existing graph to obtain a new graph, and we will enumerate the obtained graphs by the number of edges they contain. Start with an initial finite directed graph, denoted $G\left(n_{0}\right)$, with at least one node and $n_{0}$ edges. For $n=n_{0}+1, n_{0}+2, \ldots, G(n)$ will be a graph with $n$ edges and a random number $N(n)$ of nodes. If $u$ is a node in $G(n-1), D_{\text {in }}(u)$ and $D_{\text {out }}(u)$ denote the in and out degree of $u$ respectively. The graph $G(n)$ is obtained from the graph $G(n-1)$ as follows.

- With probability $\alpha$ we append to $G(n-1)$ a new node $v$ and an edge leading from $v$ to an existing node $w$ in $G(n-1)$ (denoted $v \mapsto w)$. The existing node $w$ in $G(n-1)$ is chosen with probability depending on its in-degree:

$$
p(w \text { is chosen })=\frac{D_{\text {in }}(w)+\delta_{\text {in }}}{n-1+\delta_{\mathrm{in} n} N(n-1)} .
$$

- With probability $\beta$ we only append to $G(n-1)$ a directed edge $v \mapsto w$ between two existing nodes $v$ and $w$ of $G(n-1)$. The existing nodes $v, w$ are chosen independently from the nodes of $G(n-1)$ with probabilities

$$
p(v \text { is chosen })=\frac{D_{\text {out }}(v)+\delta_{\text {out }}}{n-1+\delta_{\text {out }} N(n-1)}, \quad p(w \text { is chosen })=\frac{D_{\text {in }}(w)+\delta_{\text {in }}}{n-1+\delta_{\text {in }} N(n-1)} .
$$


- With probability $\gamma$ we append to $G(n-1)$ a new node $w$ and an edge $v \mapsto w$ leading from the existing node $v$ in $G(n-1)$ to the new node $w$. The existing node $v$ in $G(n-1)$ is chosen with probability

$$
p(v \text { is chosen })=\frac{D_{\text {out }}(v)+\delta_{\text {out }}}{n-1+\delta_{\text {out }} N(n-1)} .
$$

If either $\delta_{\text {in }}=0$, or $\delta_{\text {out }}=0$, we must have $n_{0} \geq 1$ for the initial steps of the algorithm to make sense.

For $i, j=0,1,2, \ldots$ and $n \geq n_{0}$, let $N_{i j}(n)$ be the (random) number of nodes in $G(n)$ with indegree $i$ and out-degree $j$. Theorem 3.2 in Bollobás et al. (2003) shows that there are nonrandom constants $\left(f_{i j}\right)$ such that

$$
\lim _{n \rightarrow \infty} \frac{N_{i j}(n)}{n}=f_{i j} \text { a.s. for } i, j=0,1,2, \ldots
$$

Clearly, $f_{00}=0$. Since we obviously have

$$
\lim _{n \rightarrow \infty} \frac{N(n)}{n}=1-\beta \text { a.s., }
$$

we see that the empirical joint in- and out-degree distribution in the sequence $\{G(n)\}$ of growing random graphs has as a nonrandom limit the probability distribution

$$
\lim _{n \rightarrow \infty} \frac{N_{i j}(n)}{N(n)}=\frac{f_{i j}}{1-\beta}:=p_{i j} \text { a.s. for } i, j=0,1,2, \ldots
$$

In Bollobás et al. (2003) it was shown that the limiting degree distribution $\left(p_{i j}\right)$ has, marginally, regularly varying (in fact, power-like) tails. Specifically, Theorem 3.1 ibid. shows that for some finite positive constants $C_{\text {in }}$ and $C_{\text {out }}$ we have

$$
\begin{aligned}
p_{i}(\text { in }) & :=\sum_{j=0}^{\infty} p_{i j} \sim C_{\mathrm{i} n} i^{-\alpha_{\mathrm{i} n}} \text { as } i \rightarrow \infty, \text { as long as } \alpha \delta_{\mathrm{in}}+\gamma>0, \\
p_{j}(\text { out }) & :=\sum_{i=0}^{\infty} p_{i j} \sim C_{\mathrm{out}} j^{-\alpha_{\text {out }}} \text { as } j \rightarrow \infty, \text { as long as } \gamma \delta_{\mathrm{out}}+\alpha>0 .
\end{aligned}
$$

Here

$$
\alpha_{\mathrm{i} n}=1+\frac{1+\delta_{\mathrm{in}}(\alpha+\gamma)}{\alpha+\beta}, \quad \alpha_{\mathrm{out}}=1+\frac{1+\delta_{\mathrm{out}}(\alpha+\gamma)}{\gamma+\beta} .
$$

In fact, the limiting degree distribution $\left(p_{i j}\right)$ in (4.2) generates a distribution that has jointly nonstandard regularly varying tails and the limit measure of regular variation has a density as shown in Samorodnitsky et al. (2014).

4.2. Notation and results summary. We summarize results and notation for the preferential attachment model from Samorodnitsky et al. (2014).

$$
\begin{aligned}
& c_{1}=\frac{\alpha+\beta}{1+\delta_{\text {in }}(\alpha+\gamma)}=\frac{1}{\alpha_{\text {in }}-1}, \quad c_{2}=\frac{\beta+\gamma}{1+\delta_{\text {out }}(\alpha+\gamma)},=\frac{1}{\alpha_{\text {out }}-1} \\
& a=c_{2} / c_{1} .
\end{aligned}
$$


We developed an explicit formula for the joint generating function of in- and out-degree. The joint generating function of $\left\{p_{i j}\right\}$ in (4.2),

$$
\varphi(x, y)=\sum_{i=0}^{\infty} \sum_{j=0}^{\infty} x^{i} y^{j} p_{i j}, 0 \leq x, y \leq 1,
$$

satisfies a partial differential equation that, when solved, yields

$$
\varphi(x, y)=\frac{\gamma}{\alpha+\gamma} x \varphi_{1}(x, y)+\frac{\alpha}{\alpha+\gamma} y \varphi_{2}(x, y),
$$

with

$$
\begin{aligned}
& \varphi_{1}(x, y)=c_{1}^{-1} \int_{1}^{\infty} z^{-\left(1+1 / c_{1}\right)}(x+(1-x) z)^{-\left(\delta_{\mathrm{i} n}+1\right)}\left(y+(1-y) z^{a}\right)^{-\delta_{\mathrm{out}}} d z, \\
& \varphi_{2}(x, y)=c_{1}^{-1} \int_{1}^{\infty} z^{-\left(1+1 / c_{1}\right)}(x+(1-x) z)^{-\delta_{\mathrm{i} n}}\left(y+(1-y) z^{a}\right)^{-\left(\delta_{\mathrm{ou} t}+1\right)} d z
\end{aligned}
$$

for $0 \leq x, y \leq 1$. Each of $\varphi_{1}, \varphi_{2}$ is the joint generating function of a pair of nonnegative integervalued random variables; that is, on some probability space we can find nonnegative integer-valued random variables $X_{j}, Y_{j}, j=1,2$ such that

$$
\varphi_{j}(x, y)=E\left(x^{X_{j}} y^{Y_{j}}\right), 0 \leq x, y \leq 1, j=1,2 .
$$

If $(I, O)$ is a random vector with generating function (4.8),

$$
\varphi(x, y)=E\left(x^{I} y^{O}\right)
$$

we can represent the distribution of $(I, O)$ as

$$
(I, 0) \stackrel{d}{=} B\left(1+X_{1}, Y_{1}\right)+(1-B)\left(X_{2}, 1+Y_{2}\right),
$$

where $B$ is a Bernoulli switching variable independent of $X_{j}, Y_{j}, j=1,2$ with

$$
P[B=1]=1-P[B=0]=\frac{\gamma}{\alpha+\gamma} .
$$

The explicit structure and form in (4.8), (4.9) and (4.10) allowed analysis of the asymptotic multivariate power law structure performed in Samorodnitsky et al. (2014). Absent such structure, if all one has is the joint generating function, one would have to rely on Tauberian analysis of the transform. We show how the material in Section 2 is applicable.

4.3. Joint regular variation of the distribution of in-degree and out-degree. In this section we apply the Tauberian theorem of Section 3 to the joint generating function $\varphi$ of the limiting distribution of in- and out-degree given in (4.8), (4.9) and (4.10) to prove the nonstandard joint regular variation of in- and out-degree and obtain an expression for the density of the tail measure.

The next Theorem 3 shows that each of the random vectors $\left(X_{j}, Y_{j}\right), j=1,2$, has a nonstandard regularly varying distribution. The decomposition (4.8) allows us to deduce the nonstandard joint regular variation of $(I, O)$, the in-degree and out-degree.

Theorem 3. Assume that $\delta_{\text {in }}>0$ and $\delta_{\text {out }}>0$, and let $\alpha_{\text {in }}$ and $\alpha_{\text {out }}$ be given by (4.4). For each $j=1,2$ there is a Radon measure $V_{j} \in M_{+}\left([0, \infty]^{2} \backslash\{\mathbf{0}\}\right)$ such that as $h \rightarrow \infty$,

$$
h P\left[\left(h^{-1 /\left(\alpha_{\text {in }}-1\right)} X_{j}, h^{-1 /\left(\alpha_{\text {out }}-1\right)} Y_{j}\right) \in \cdot\right] \stackrel{v}{\rightarrow} V_{j}(\cdot),
$$


TAUBERIAN THEORY AND PREFERENTIAL ATTACHMENT

vaguely in $M_{+}\left([0, \infty]^{2} \backslash\{\mathbf{0}\}\right)$. Furthermore, $V_{1}$ and $V_{2}$ concentrate on $(0, \infty)^{2}$ and have Lebesgue densities $f_{1}, f_{2}$ given by,

$$
f_{1}(x, y)=c_{1}^{-1}\left(\Gamma\left(\delta_{\text {in }}+1\right) \Gamma\left(\delta_{\text {out }}\right)\right)^{-1} x^{\delta_{\text {in }}} y^{\delta_{\text {out }}-1} \int_{0}^{\infty} z^{-\left(2+1 / c_{1}+\delta_{\text {in }}+a \delta_{\text {out }}\right)} e^{-\left(x / z+y / z^{a}\right)} d z,
$$

and

$$
f_{2}(x, y)=c_{1}^{-1}\left(\Gamma\left(\delta_{\text {in }}\right) \Gamma\left(\delta_{\text {out }}+1\right)\right)^{-1} x^{\delta_{\text {in }}-1} y^{\delta_{\text {out }}} \int_{0}^{\infty} z^{-\left(1+a+1 / c_{1}+\delta_{\text {in }}+a \delta_{\text {out }}\right)} e^{-\left(x / z+y / z^{a}\right)} d z
$$

The random vector $(I, O)$ with joint mass function $\left\{p_{i j}\right\}$ in (4.2) satisfies as $h \rightarrow \infty$,

$$
h P\left[\left(h^{-1 /\left(\alpha_{\text {in }}-1\right)} I, h^{-1 /\left(\alpha_{\text {out }}-1\right)} O\right) \in \cdot\right] \stackrel{v}{\rightarrow} \frac{\gamma}{\alpha+\gamma} V_{1}(\cdot)+\frac{\alpha}{\alpha+\gamma} V_{2}(\cdot),
$$

vaguely in $M_{+}\left([0, \infty]^{2} \backslash\{\mathbf{0}\}\right)$.

Proof. It is enough to prove (4.12), (4.13) and (4.14). We treat the case $j=1$. The case $j=2$ is analogous. Since $\varphi_{1}(x, y)$ is the generating function of a probability mass function, simply converting $\varphi_{1}(x, y)$ into a Laplace transform will not yield the Laplace transform of an infinite measure $U$ as required by the previous section. So we first modify the generating function.

Choose and fix a positive integer $k>\alpha_{\mathrm{in}}-1$. This choice of $k$ guarantees $E\left(X_{1}^{k}\right)=\infty$. Denote

$$
\psi(x, y)=\frac{\partial^{k} \varphi_{1}}{\partial x^{k}}(x, y), 0<x, y<1,
$$

so that the function $\psi$ can be written in the form

$$
\psi(x, y)=\sum_{i=0}^{\infty} \sum_{j=0}^{\infty} x^{i} y^{j} m_{i j}^{(k)}, 0<x, y<1,
$$

where

$$
m_{i j}^{(k)}=\prod_{d=1}^{k}(i+d) p_{i j}^{(k)}, i, j=0,1,2, \ldots,
$$

and $\left(p_{i j}^{(k)}\right)$ is the joint probability mass function of the random vector $\left(X_{1}-k, Y_{1}\right)$. Let $U(\cdot)=$ $\sum_{i, j} m_{i j}^{(k)} \epsilon_{(i, j)}(\cdot)$ be the infinite Radon measure on $[0, \infty)^{2}$ concentrating on $(\{0,1,2, \ldots\})^{2}$ that puts mass $m_{i j}^{(k)}$ at $(i, j)$. To verify this measure is infinite, observe

$$
\sum_{i, j} m_{i j}^{(k)}=\sum_{l=0}^{\infty} \prod_{p=1}^{k}(p+l) P\left[X_{1}=l+k\right]
$$

and since $\prod_{p=1}^{k}(p+l) \sim(l+k)^{k}$ as $l \rightarrow \infty$ and $E\left(X_{1}^{k}\right)=\infty$, we have $\sum_{i, j} m_{i j}^{(k)}$ diverges.

Using Proposition 2, we show that the measure $U$ is regularly varying: As $h \rightarrow \infty$, we show,

$$
\frac{1}{h} U\left\{(i, j):\left(h^{-1 /\left(k-\alpha_{\mathrm{i} n}+1\right)} i, h^{-\left(\alpha_{\mathrm{in} n}-1\right) /\left(\left(\alpha_{\mathrm{out}}-1\right)\left(k-\alpha_{\mathrm{in}}+1\right)\right)} j\right) \in \cdot\right\} \stackrel{v}{\rightarrow} V_{1, k}(\cdot)
$$

vaguely in $M_{+}\left([0, \infty)^{2}\right)$, where the Radon measure $V_{1, k}$ concentrates on $(0, \infty)^{2}$ with density

$$
f_{1, k}(x, y)=c_{1}^{-1}\left(\Gamma\left(\delta_{\mathrm{i} n}+1\right) \Gamma\left(\delta_{\mathrm{out}}\right)\right)^{-1} x^{\delta_{\mathrm{i} n}+k} y^{\delta_{\mathrm{out}}-1} \int_{0}^{\infty} z^{-\left(2+1 / c_{1}+\delta_{\mathrm{i} n}+a \delta_{\mathrm{out}}\right)} e^{-\left(x / z+y / z^{a}\right)} d z
$$


To this end, using the form of $\phi_{1}$ in (4.9), we write the function $\psi$ in (4.15) explicitly as

$$
\begin{aligned}
\psi(x, y) & =c_{1}^{-1} \prod_{i=1}^{k}\left(\delta_{\mathrm{i} n}+i\right) \int_{1}^{\infty} z^{-\left(1+1 / c_{1}\right)}(z-1)^{k}(x+(1-x) z)^{-\left(\delta_{\mathrm{i} n}+k+1\right)}\left(y+(1-y) z^{a}\right)^{-\delta_{\text {out }}} d z \\
& :=c_{1}^{-1} \prod_{i=1}^{k}\left(\delta_{\mathrm{i} n}+i\right) \tilde{\psi}(x, y) .
\end{aligned}
$$

We switch from generating functions to Laplace transforms by replacing $(x, y)$ with $e^{-\boldsymbol{\lambda}}=\left(e^{-\lambda_{1}}, e^{-\lambda_{2}}\right)$ and then consider regular variation of the resulting Laplace transform. For fixed $\lambda_{1}>0, \lambda_{2}>0$ elementary calculations show that, as $h \rightarrow \infty$,

$$
\begin{aligned}
h^{-1} \tilde{\psi} & \left(e^{-\lambda_{1} h^{-1 /\left(k-\alpha_{\mathrm{i} n}+1\right)}}, e^{-\lambda_{2} h^{-\left(\alpha_{\mathrm{i} n}-1\right) /\left(\alpha_{\mathrm{out}}-1\right)\left(k-\alpha_{\mathrm{in}}+1\right)}}\right) \\
& \sim h^{-1} \int_{1}^{\infty} z^{k-1-1 / c_{1}}\left(1+z \lambda_{1} h^{-1 /\left(k-\alpha_{\mathrm{in}}+1\right)}\right)^{-\left(\delta_{\mathrm{i} n}+k+1\right)} \\
& \times\left(1+z^{a} \lambda_{2} h^{-\left(\alpha_{\mathrm{i} n}-1\right) /\left(\left(\alpha_{\mathrm{out}}-1\right)\left(k-\alpha_{\mathrm{in}}+1\right)\right)}\right)^{-\delta_{\mathrm{out}}} d z \\
& =\int_{h^{-1 /\left(k-\alpha_{\mathrm{in}}+1\right)}}^{\infty} z^{k-1-1 / c_{1}}\left(1+z \lambda_{1}\right)^{-\left(\delta_{\mathrm{i} n}+k+1\right)}\left(1+z^{a} \lambda_{2}\right)^{-\delta_{\text {out }}} d z \\
& \rightarrow \int_{0}^{\infty} z^{k-1-1 / c_{1}}\left(1+z \lambda_{1}\right)^{-\left(\delta_{\mathrm{i} n}+k+1\right)}\left(1+z^{a} \lambda_{2}\right)^{-\delta_{\text {out }}} d z .
\end{aligned}
$$

We conclude that for any $\lambda_{1}>0, \lambda_{2}>0$, as $h \rightarrow \infty$,

$$
\begin{aligned}
h^{-1} \hat{U} & \left(\lambda_{1} h^{-1 /\left(k-\alpha_{\mathrm{in} n}+1\right)}, \lambda_{2} h^{-\left(\alpha_{\mathrm{in} n}-1\right) /\left(\left(\alpha_{\mathrm{out}}-1\right)\left(k-\alpha_{\mathrm{in}}+1\right)\right)}\right) \\
& =h^{-1} \psi\left(e^{-\lambda_{1} h^{-1 /\left(k-\alpha_{\mathrm{i} n}+1\right)}}, e^{\left.-\lambda_{2} h^{-\left(\alpha_{\mathrm{i} n}-1\right) /\left(\left(\alpha_{\mathrm{out}}-1\right)\left(k-\alpha_{\mathrm{in}}+1\right)\right)}\right)}\right. \\
& \rightarrow c_{1}^{-1} \prod_{i=1}^{k}\left(\delta_{\mathrm{i} n}+i\right) \int_{0}^{\infty} z^{k-1-1 / c_{1}}\left(1+z \lambda_{1}\right)^{-\left(\delta_{\mathrm{i} n}+k+1\right)}\left(1+z^{a} \lambda_{2}\right)^{-\delta_{\mathrm{out}}} d z \\
& =\int_{[0, \infty)^{2}} e^{-\left(\lambda_{1} v_{1}+\lambda_{2} v_{2}\right)} V_{1, k}\left(d v_{1}, d v_{2}\right),
\end{aligned}
$$

where the measure $V_{1, k}$ concentrates on $(0, \infty)^{2}$ and has density

$$
f_{1, k}(x, y)=c_{1}^{-1} \prod_{i=1}^{k}\left(\delta_{\mathrm{in}}+i\right) \int_{0}^{\infty} z^{k-1-1 / c_{1}} \frac{x^{\delta_{\mathrm{i} n}+k} z^{-\left(\delta_{\mathrm{i} n}+k+1\right)}}{\Gamma\left(\delta_{\mathrm{i} n}+k+1\right)} e^{-x / z} \frac{y^{\delta_{\mathrm{out}}-1}\left(z^{a}\right)^{-\delta_{\mathrm{out}}}}{\Gamma\left(\delta_{\mathrm{out}}\right)} e^{-y / z^{a}} d z,
$$

given by (4.17).

The claim (4.16) now follows from (4.18) and the Tauberian result in Proposition 2 provided we check that the measure $U$ satisfies condition (3.9) of that result so we must check with

$$
\boldsymbol{b}(h)=\left(h^{1 /\left(k-\alpha_{\mathrm{i} n}+1\right)}, h^{\left(\alpha_{\mathrm{i} n}-1\right) /\left(\left(\alpha_{\mathrm{out}}-1\right)\left(k-\alpha_{\mathrm{i} n}+1\right)\right)}\right)
$$

that

$$
\lim _{y \rightarrow \infty} \limsup _{h \rightarrow \infty} \int_{\left[v_{1}>y\right] \cup\left[v_{2}>y\right]} e^{-\boldsymbol{\lambda}^{\prime} \boldsymbol{v}} h^{-1} U(\boldsymbol{b}(h) d \boldsymbol{v})=0 .
$$


Considering the definition of $U(\cdot)$ the integral in (4.19) becomes, after a change of variable $s_{i}=$ $b_{i}(h) v_{i}$,

$$
\begin{aligned}
\int_{\left[s_{1}>b_{1}(h) y\right] \cup\left[s_{2}>b_{2}(h) y\right]} e^{-\left(\lambda_{1} s_{1} / b_{1}(h)+\lambda_{2} s_{2} / b_{2}(h)\right)} h^{-1} U(d s) \\
=\sum_{\left[i>b_{1}(h) y\right] \cup\left[j>b_{2}(h) y\right]} e^{-\left(\lambda_{1} i / b_{1}(h)+\lambda_{2} j / b_{2}(h)\right)} h^{-1} m_{i j}^{(k)} \\
=\sum_{\left[i>b_{1}(h) y\right] \cup\left[j>b_{2}(h) y\right]} e^{-\left(\lambda_{1} i / b_{1}(h)+\lambda_{2} j / b_{2}(h)\right)} h^{-1} \prod_{d=1}^{k}(i+d) p_{i j}^{(k)} \\
\leq \sum_{i>b_{1}(h) y}+\sum_{j>b_{2}(h) y} .
\end{aligned}
$$

Notice that

$$
\begin{aligned}
& \sum_{i>b_{1}(h) y} e^{-\left(\lambda_{1} i / b_{1}(h)+\lambda_{2} j / b_{2}(h)\right)} h^{-1} \prod_{d=1}^{k}(i+d) p_{i j}^{(k)} \\
\leq & \sum_{i>b_{1}(h) y} e^{-\left(\lambda_{1} i / b_{1}(h)\right)} h^{-1} \sum_{j} \prod_{d=1}^{k}(i+d) p_{i j}^{(k)} \\
= & \sum_{i>b_{1}(h) y} e^{-\left(\lambda_{1} i / b_{1}(h)\right)} h^{-1} \prod_{d=1}^{k}(i+d) p_{i+k}(i n)
\end{aligned}
$$

using the notation from (4.3). Set $u_{i}=\prod_{d=1}^{k}(i+d) p_{i+k}(i n)$ so from (4.3) $u_{i} \sim C_{\text {in }} i^{k-\alpha_{\mathrm{in}}}$. Letting $C$ be a finite constant, the sum on the previous line is bounded by

$$
\begin{aligned}
& C \sum_{i>b_{1}(h) y} e^{-\left(\lambda_{1} i / b_{1}(h)\right)} h^{-1} i^{k-\alpha_{\mathrm{i} n}} \\
& \sim C h^{-1} \int_{b_{1}(h) y}^{\infty} e^{-\left(\lambda_{1} x / b_{1}(h)\right)} x^{k-\alpha_{\mathrm{i} n}} d x \\
& \rightarrow C \int_{y}^{\infty} e^{-\lambda_{1} x} x^{k-\alpha_{\mathrm{i} n}} d x, \quad(h \rightarrow \infty), \\
& \rightarrow 0 \quad(y \rightarrow \infty) .
\end{aligned}
$$

In particular, given $\varepsilon>0$, there is $\theta_{\varepsilon} \in(0, \infty)$ such that for all $h$ large enough,

$$
\sum_{i>b_{1}(h) \theta_{\varepsilon}} e^{-\left(\lambda_{1} i / b_{1}(h)+\lambda_{2} j / b_{2}(h)\right)} h^{-1} \prod_{d=1}^{k}(i+d) p_{i j}^{(k)} \leq \varepsilon .
$$

For such $h$,

Further, for some positive constant $C$,

$$
\sum_{j>b_{2}(h) y} \leq \varepsilon+\sum_{i \leq b_{1}(h) \theta_{\varepsilon}, j>b_{2}(h) y} .
$$

$$
\sum_{i \leq b_{1}(h) \theta_{\varepsilon}, j>b_{2}(h) y} e^{-\left(\lambda_{1} i / b_{1}(h)+\lambda_{2} j / b_{2}(h)\right)} h^{-1} \prod_{d=1}^{k}(i+d) p_{i j}^{(k)}
$$




$$
\begin{aligned}
& \leq C \sum_{i \leq b_{1}(h) \theta_{\varepsilon}, j>b_{2}(h) y} h^{-1} i^{k} p_{i j}^{(k)} \\
& \leq C \theta_{\varepsilon}^{k} h^{-1} b_{1}(h)^{k} \sum_{j>b_{2}(h) y} p_{i j}^{(k)} \\
& =C \theta_{\varepsilon}^{k} h^{\left(\alpha_{\mathrm{i} n}-1\right) /\left(k-\alpha_{\mathrm{i} n}+1\right)} \sum_{j>b_{2}(h) y} p_{j}(\text { out }) \\
& \sim\left(C C_{\text {out }} \theta_{\varepsilon}^{k} /\left(\alpha_{\text {out }}-1\right)\right) h^{\left(\alpha_{\mathrm{i} n}-1\right) /\left(k-\alpha_{\mathrm{i} n}+1\right)}\left(b_{2}(h) y\right)^{-\left(\alpha_{\mathrm{out}}-1\right)} \\
& =\left(C C_{\text {out }} \theta_{\varepsilon}^{k} /\left(\alpha_{\mathrm{out}}-1\right)\right) y^{-\left(\alpha_{\mathrm{out}}-1\right)} \\
& \rightarrow 0 \quad(y \rightarrow \infty)
\end{aligned}
$$

by the Karamata theorem, using the notation from (4.3).

Letting $\varepsilon \rightarrow 0$ we see that we have verified that the measure $U$ satisfies condition (3.9) and that (4.16) holds and we are now ready to prove (4.12). Let $\mu^{(k)}=\sum_{i, j} p_{i j}^{(k)} \epsilon_{(i, j)}$ be the probability measure concentrating on $(\{0,1,2, \ldots\})^{2}$ that puts mass $p_{i j}^{(k)}$ at $(i, j)$. For (4.12), it is enough to prove that for any $a, b>0$,

$$
h \int_{h^{1 /\left(\alpha_{\mathrm{in}}-1\right)} a}^{\infty} \int_{h^{1 /\left(\alpha_{\mathrm{out}}-1\right) b}}^{\infty} \mu^{(k)}(d x, d y) \rightarrow \int_{a}^{\infty} \int_{b}^{\infty} f_{1}(x, y) d x d y
$$

as $h \rightarrow \infty$, with $f_{1}$ given by (4.13). Indeed, by Theorem 3.2 in Bollobás et al. (2003), the conditional distributions of the random vector $(I, O)$ are also regular varying with exponents of regular variation strictly larger than those of the marginal distributions. Therefore, one can trivially add the axes $\{x=0, y>0\}$ and $\{x>0, y=0\}$ to the convergence in (4.20) which yields

$$
h P\left[\left(h^{-1 /\left(\alpha_{\mathrm{i} n}-1\right)}\left(X_{1}-k\right), h^{-1 /\left(\alpha_{\mathrm{out}}-1\right)} Y_{1}\right) \in \cdot\right] \stackrel{v}{\rightarrow} V_{1}(\cdot),
$$

which is equivalent to (4.12) with $j=1$.

It remains, therefore, to prove (4.20). Fix $M>\max (a, b)$. Since

$$
\mu^{(k)}(d x, d y)=\frac{U(d x, d y)}{\prod_{d=1}^{k}(x+d)},
$$

we have, as $h \rightarrow \infty$,

$$
\begin{gathered}
h \int_{h^{1 /\left(\alpha_{\mathrm{i} n}-1\right)} a}^{h^{1 /\left(\alpha_{\mathrm{i} n}-1\right)} M} \int_{h^{1 /\left(\alpha_{\mathrm{out}}-1\right) b}}^{h^{1 /\left(\alpha_{\mathrm{out}}-1\right)} M} \mu^{(k)}(d x, d y) \sim h \int_{h^{1 /\left(\alpha_{\mathrm{i} n}-1\right)} a}^{h^{1 /\left(\alpha_{\mathrm{i} n}-1\right)} M} \int_{h^{1 /\left(\alpha_{\mathrm{out}}-1\right) b}}^{h^{1 /\left(\alpha_{\mathrm{out}}-1\right)} M} x^{-k} U(d x, d y) \\
=h^{1-k /\left(\alpha_{\mathrm{out}}-1\right)} \int_{a}^{M} \int_{b}^{M} x^{-k} U\left(d h^{1 /\left(\alpha_{\mathrm{i} n}-1\right)} x, d h^{1 /\left(\alpha_{\mathrm{out}}-1\right)} y\right) .
\end{gathered}
$$

Denoting $m_{h}=h^{k /\left(\alpha_{\mathrm{out}}-1\right)-1}$, we can write the above as

$$
\begin{aligned}
& =\frac{1}{m_{h}} \int_{a}^{M} \int_{b}^{M} x^{-k} U\left(m_{h}^{1 /\left(k-\alpha_{\mathrm{in}}+1\right)} d x, m_{h}^{\left(\alpha_{\mathrm{in} n}-1\right) /\left(\left(\alpha_{\mathrm{out}}-1\right)\left(k-\alpha_{\mathrm{i} n}+1\right)\right)} d y\right) \\
& \rightarrow \int_{a}^{M} \int_{b}^{M} x^{-k} f_{1, k}(x, y) d x d y
\end{aligned}
$$

as $h \rightarrow \infty$ by (4.16). Since

$$
f_{1}(x, y)=x^{-k} f_{1, k}(x, y), 0<x, y<1
$$


the statement (4.12) with $j=1$ follows, because by (4.3) and (4.8),

$$
\begin{aligned}
& \limsup _{h \rightarrow \infty} h\left[\int_{h^{1 /\left(\alpha_{\mathrm{i} n}-1\right)} M}^{\infty} \int_{0}^{\infty} \mu^{(k)}(d x, d y)+\int_{0}^{\infty} \int_{h^{1 /\left(\alpha_{\mathrm{out}}-1\right)} M} \mu^{(k)}(d x, d y)\right] \\
& \quad \leq \limsup _{h \rightarrow \infty} h P\left(X_{1}>h^{1 /\left(\alpha_{\mathrm{i} n}-1\right)} M+k\right)+\underset{h \rightarrow \infty}{\limsup } h P\left(Y_{1}>h^{1 /\left(\alpha_{\mathrm{out}}-1\right)} M\right) \\
& \quad \leq \frac{\alpha+\gamma}{\gamma} \frac{C_{\mathrm{i} n}}{\alpha_{\mathrm{i} n}-1} M^{-\left(\alpha_{\mathrm{i} n}-1\right)}+\frac{\alpha+\gamma}{\alpha} \frac{C_{\mathrm{out}}}{\alpha_{\text {out }}-1} M^{-\left(\alpha_{\mathrm{out}}-1\right)},
\end{aligned}
$$

and one only needs to let $M \rightarrow \infty$.

As mentioned before, the case of (4.12) with $j=2$ is analogous.

\section{ACKnOWLedgment}

We appreciate several helpful and informative conversations with Don Towsley and Bo Jiang of the University of Massachusetts.

\section{REFERENCES}

N. Bingham, C. Goldie and J. Teugels (1987): Regular Variation. Cambridge University Press, Cambridge.

B. Bollobás, C. Borgs, J. Chayes and O. Riordan (2003): Directed scale-free graphs. In Proceedings of the Fourteenth Annual ACM-SIAM Symposium on Discrete Algorithms (Baltimore, 2003). ACM, New York, pp. 132-139.

W. Feller (1971): An Introduction to Probability Theory and its Applications, volume 2. Wiley, New York, 2nd edition.

P. Krapivsky and S. Redner (2001): Organization of growing random networks. Physical Review E 63:066123:1-14.

S. RESNICK (1991): Point processes and Tauberian theory. Math. Sci. 16:83-106.

S. Resnick (2007): Heavy-Tail Phenomena: Probabilistic and Statistical Modeling. Springer, New York.

G. Samorodnitsky, S. Resnick, D. Towsley, R. Davis, A. Willis and P. Wan (2014): Nonstandard regular variation of in-degree and out-degree in the preferential attachment model. ArXiv e-prints http://arxiv.org/pdf/1405.4882.pdf.

U. StadtmülleR (1981): A refined Tauberian theorem for Laplace transforms in dimension $d>1$. J. Reine Angew. Math. 328:72-83.

U. Stadtmüller and R. Trautner (1979): Tauberian theorems for Laplace transforms. J. Reine Angew. Math. 311/312:283-290.

U. Stadtmüller and R. Trautner (1981): Tauberian theorems for Laplace transforms in dimension $D>1$. J. Reine Angew. Math. 323:127-138.

A. Stam (1977): Regular variation in $\mathbb{R}_{+}^{d}$ and the Abel-Tauber theorem. Technical Report, unpublished, Mathematisch Instituut, Rijksuniversiteit Groningen.

A. Yakimiv (2005): Probabilistic Applications of Tauberian Theorems. Modern Probability and Statistics. VSP, Leiden, The Netherlands. 
School of Operations Research and Information Engineering, and Department of Statistical Science, Cornell University, Ithaca, NY 14853

E-mail address: sir1@cornell.edu

School of Operations Research and Information Engineering, and Department of Statistical SciEnce, Cornell University, IthacA, NY 14853

E-mail address: gs18@cornell.edu 